\title{
Mathematical and Numerical Analysis of a Robust and Efficient Grid Deformation Method in the Finite Element Context
}

\author{
Matthias Grajewski*† $\quad$ Michael Köster ${ }^{\ddagger} \quad$ Stefan Turek $\S$
}

April 22, 2008

\begin{abstract}
Among a variety of grid deformation methods, the method proposed by Liao $[4,6,18]$ is one of the most favourables, because it prevents mesh tangling and offers precise control over the element volumes. Its numerical realisation only requires solving a Poisson problem and a system of fully decoupled initial value problems. Many other deformation methods in contrast involve the solution of complex nonlinear PDEs. In this article, we introduce a generalisation of Liao's method which allows for generating a desired mesh size distribution for quite arbitrary grids without giving rise to mesh tangling. We elaborate on its numerical realisation and prove the convergence of our method. Our results are confirmed by numerical experiments.
\end{abstract}

Keywords: mesh generation, deformation method, a posteriori error estimation, mesh adaption

AMS classification: 65N15, 65N30, 76D05, 76D55

\section{Introduction}

Grid deformation, i.e. the redistribution of the mesh points of a given grid preserving the mesh topology, is a topic of research since many years and, consequently, a variety of methods is available today (see [5, 7, 10, 24] among many others). Most of the grid deformation approaches can be divided into two groups: the group of static methods and the group of dynamic approaches. Static methods obtain the mapping to the deformed grid by minimising certain functionals which usually leads to the (often difficult and expensive) solution of non-linear PDEs. In contrast to this, dynamic methods (e.g. $[9,17])$ use time stepping or pseudo-time stepping approaches to construct the desired transformation. Being a member of the latter group, the method developed by Liao and

\footnotetext{
* corresponding author

${ }^{\dagger}$ Institute of Applied Mathematics, Dortmund University of Technology, Vogelpothsweg 87, D-44227 Dortmund, Germany, matthias.grajewski@mathematik.tu-dortmund.de

${ }^{\ddagger}$ Institute of Applied Mathematics, Dortmund University of Technology, Vogelpothsweg 87, D-44227 Dortmund, Germany, michael.koester@mathematik.tu-dortmund.de

${ }^{\S}$ Institute of Applied Mathematics, Dortmund University of Technology, Vogelpothsweg 87, D-44227 Dortmund, Germany, ture@featflow.de
} 
his coworkers, based on the work of Moser [11], requires the solution of a single Poisson equation and a decoupled system of initial value problems (IVPs) only. Furthermore, it has been shown that tangled elements cannot occur [20].

There are a couple of reasons to investigate and to apply a grid deformation method in order to adapt a given computational mesh ( $r$-adaptivity):

In many applications, local and anisotropic phenomena like shock fronts occur. It was shown $[1,12]$ that by anisotropic refinement and alignment according to such phenomena, the accuracy of the calculation can be vastly improved. Refining the region of a shock by using hanging nodes solely ( $h$-adaptivity) may suffer from the fact that the given grid is not aligned with the shock. In contrast, $r$-adaptive methods may provide additional flexibility which allows for aligning the grid cells to the shock.

In FEM simulation it has turned out that grid adaptivity governed by a posteriori error estimation is mandatory for reliable and efficient computations. The widely used method of grid adaptation by allowing hanging nodes on element level, however, requires data structures which rely on the extensive usage of indirect adressing. This is necessary to handle the unstructured grids emerging from the adaptation procdure. Recent research [2] has shown that in typical adaptive FEM codes using this method of grid adaptation only a small fraction of the available processor performance of several GFlop/s can be typically used. On the other hand, by using local generalised tensor product meshes and thereby avoiding indirect adressing a very significant speed up can be achieved. This has been successfully implemented in our new FEM package FEAST [2]. In this context, grid deformation is an ideal tool for grid adaptation, as it preserves the local generalised tensor product structure while providing flexibility in adaptation comparable to elementwise $h$-adaptivity.

In this article, we proceed as follows: In the next section, we describe in detail our deformation method and prove its basic properties. In section 3, the focus is placed on convergence analysis of the grid deformation method. We present numerical experiments supporting our theoretical results in section 4 .

\section{Description of our Deformation Method}

We first introduce some notations. A computational domain $\Omega \subset \mathbb{R}^{2}$ is triangulated by a mesh $\mathbb{T}$ consisting of $N E L$ quadrilateral elements $T$ of size $h_{T}$. We denote the set of vertices by $\mathcal{V}$ and the set of edges by $\mathcal{E}$. The mesh is supposed to be conforming, i.e. no hanging nodes are allowed. The area of an element $T$ is denoted by $m(T)$. For the common Lebesgue and Sobolev spaces on a domain $D$ we use the abbreviations $L^{2}(D)$ and $H^{k}(D)$. In the special case $D=\Omega$, we write $L^{2}$ and $H^{k}$ instead. The function space of $k$-fold continuously differentiable functions is referenced by $\mathcal{C}^{k}(D)$; for an interval $I$, $\mathcal{C}^{k, \alpha}(I), 0<\alpha<1$, denotes the space of functions with Hölder-continuous $k$ th derivatives. A domain is said to have an $\mathcal{C}^{k, \alpha}$-smooth boundary if the boundary can be parameterised by a function in $\mathcal{C}^{k, \alpha}(I)$. The Jacobian matrix of a smooth mapping $\Phi: \Omega \rightarrow \Omega$ is denoted by $J \Phi$, its determinant by $|J \Phi|$.

To formalise the deformation process, we introduce a weighting function $g \in \mathcal{C}^{1}(\bar{\Omega})$ and a monitor function $f \in \mathcal{C}^{1}(\bar{\Omega})$. Both functions must be strictly positive in $\bar{\Omega}$. The reason why $f$ is called monitor function will become clear below.

The theoretical background of our approach - like Liao's approach $[4,6,18,19]-$ is based on Moser's work [11]. The aim of the numerical grid deformation algorithm 
described below is to construct a bijective transformation $\Phi: \Omega \rightarrow \Omega$ which satisfies

$$
g(x)|J \Phi(x)|=f(\Phi(x)), \quad x \in \Omega
$$

as well as

$$
\Phi: \partial \Omega \rightarrow \partial \Omega .
$$

If such a transformation $\Phi$ has been found, the new coordinates $\xi$ of a grid point $x$ are computed by

$$
\xi:=\Phi(x)
$$

Applying the area formula to an element $T$ yields

$$
m(\Phi(T)):=\int_{\Phi(T)} 1 d x=\int_{T}|J \Phi(x)| d x,
$$

and using the $1 \times 1$-Gauss quadrature rule in formula (1), we obtain

$$
g\left(x_{c}\right) \frac{m(\Phi(T))}{m(T)}=f\left(\Phi\left(x_{c}\right)\right)+\mathcal{O}(h) .
$$

Here, $x_{c}$ stands for the center of $T$. If the function $g$ represents the distribution of the element area in the mesh, i.e. $g(x)=m(T)+\mathcal{O}(h), x \in T$, then we have

$$
m(\Phi(T))=f\left(\Phi\left(x_{c}\right)\right)+\mathcal{O}(h) .
$$

Thus by prescribing the monitor function $f$, the element $T$ will get - up to a spatially fixed scaling constant - the size defined by the value of $f$ in the position of the image of $T$ in the deformed grid.

In the special case $g \equiv 1$ investigated by Liao, the monitor function $f$ determines the relative growth or shrinkage of the elements with respect to the previous mesh, i.e., the mesh on which the deformation takes place. In Liao's methods, the monitor function $f$ does in general not describe the absolute distribution of the element size in space. If and only if the starting mesh has equidistributed element sizes, the monitor function does control the absolute element size.

In our new method, considering $g$ to be the area distribution on the undeformed mesh, $f$ in contrast describes the absolute mesh size distribution of the target grid, which is clearly independent of the starting grid. Note that condition (2) ensures that boundary points can move along the boundary only.

Then, based upon $[4,6,18,19]$, the transformation $\Phi$ is computed in four steps.

1. Scale the monitor function $f$ or the area function $g$ such that

$$
\int_{\Omega} \frac{1}{f(x)} d x=\int_{\Omega} \frac{1}{g(x)} d x .
$$

For the sake of simplicity, we will assume that (4) is fulfilled from now on. Let $\tilde{f}$ and $\tilde{g}$ denote the reciprocals of the scaled functions $f$ and $g$.

2. Compute a grid-velocity vector field $v: \Omega \rightarrow \mathbb{R}^{n}$ satisfying

$$
-\operatorname{div}(v(x))=\tilde{f}(x)-\tilde{g}(x), x \in \Omega, \quad \text { and } \quad v(x) \cdot \mathfrak{n}=0, x \in \partial \Omega,
$$

with $\mathfrak{n}$ being the outer normal vector of the domain boundary $\partial \Omega$, which may consist of several boundary components. 
3. For each grid point $x$, solve the initial value problem

$$
\frac{\partial \varphi(x, t)}{\partial t}=\eta(\varphi(x, t), t), \quad 0 \leq t \leq 1, \quad \varphi(x, 0)=x
$$

with

$$
\eta(y, s):=\frac{v(y)}{s \tilde{f}(y)+(1-s) \tilde{g}(y)}, \quad y \in \Omega, s \in[0,1] .
$$

4. Define

$$
\Phi(x):=\varphi(x, 1) .
$$

The divergence equation in the second step does not provide a unique solution. Therefore, we compute $v$ by solving the pure Neumann problem

$$
-\Delta w=\tilde{f}-\tilde{g}, \quad \partial_{\mathfrak{n}} w=0 \text { on } \partial \Omega
$$

and setting $v:=\nabla w$. Doing so, we implicitly fulfil the side condition curl $\mathrm{v}=0$ which provides uniqueness.

Theorem 2.1. Let the boundary of $\Omega$ be $\mathcal{C}^{3, \alpha}$-smooth and let $f, g \in \mathcal{C}^{1}(\bar{\Omega})$ be strictly positive in $\bar{\Omega}$. Then, if the mapping $\Phi: \Omega \rightarrow \Omega$ constructed above exists, it fulfils conditions (1) and (2).

Proof: Define the auxiliary function

$$
H(x, t):=|J \varphi(x, t)|[t \tilde{f}(\varphi(x, t))+(1-t) \tilde{g}(\varphi(x, t))] .
$$

We now show $\partial_{t} H(x, t)=0$. From equation $(7)$, we have

$$
v(x)=\eta(x, t)(t \tilde{f}(x)+(1-t) \tilde{g}(x)) .
$$

Applying the chain rule and the div-Operator, we find

$$
\begin{aligned}
\operatorname{div}(v(x))= & \operatorname{div}[\eta(x, t) \cdot(t \tilde{f(x)}+(1-t) \tilde{g}(x))] \\
= & {[t \tilde{f}(x)+(1-t) \tilde{g}(x)] \operatorname{div} \eta(x, t) } \\
& +(t \nabla \tilde{f}(x)+(1-t) \nabla \tilde{g}(x), \eta(x, t)) .
\end{aligned}
$$

Therefore it follows

$$
\begin{aligned}
\operatorname{div}(v(\varphi(x, t)))= & \operatorname{div}(\eta(\varphi(x, t), t))[t \tilde{f}(\varphi(x, t))+(1-t) \tilde{g}(\varphi(x, t))] \\
& +(t \nabla \tilde{f}(\varphi(x, t))+(1-t) \nabla \tilde{g}(\varphi(x, t)), \eta(\varphi(x, t))) .
\end{aligned}
$$

Starting from the ODE (6), we have by Abel's formula

$$
\begin{aligned}
|J \varphi(x, t)| & =\exp \int_{0}^{t} \operatorname{tr}(J \eta(\varphi(x, s), s)) d s \\
& =\exp \int_{0}^{t} \operatorname{div} \eta(\varphi(x, s), s) d s
\end{aligned}
$$


and by differentiation of (11) we obtain

$$
\left.\frac{\partial}{\partial t}|J \varphi(x, t)|=|J \varphi(x, t)| \operatorname{div} \eta(\varphi(x, t), t)\right) .
$$

Therefore, we obtain

$$
\begin{aligned}
& \frac{\partial}{\partial t} H(x, t)=\left(\frac{\partial}{\partial t}|J \varphi(x, t)|\right) \cdot[t \tilde{f}(\varphi(x, t))+(1-t) \tilde{g}(\varphi(x, t))] \\
& +|J \varphi(x, t)| \cdot\left[\tilde{f}(\varphi(x, t))+t\left((\nabla \tilde{f})(\varphi(x, t)), \frac{\partial}{\partial t} \varphi(x, t)\right)\right. \\
& \left.-\tilde{g}(\varphi(x, t))+(1-t)\left((\nabla \tilde{g})(\varphi(x, t)), \frac{\partial}{\partial t} \varphi(x, t)\right)\right] \\
& \stackrel{(12)}{=}|J \varphi(x, t)| \cdot \operatorname{div} \eta(\varphi(x, t), t) \cdot[t \tilde{f}(\varphi(x, t))+(1-t) \tilde{g}(\varphi(x, t))] \\
& +|J \varphi(x, t)| \cdot[\tilde{f}(\varphi(x, t))-\tilde{g}(\varphi(x, t))+ \\
& \left.\left(t \nabla \tilde{f}(\varphi(x, t))+(1-t) \nabla \tilde{g}(\varphi(x, t)), \frac{\partial}{\partial t} \varphi(x, t)\right)\right] \\
& \stackrel{(6)}{=}|J \varphi(x, t)| \cdot[\operatorname{div} \eta(\varphi(x, t), t)[t \tilde{f}(\varphi(x, t))+(1-t) \tilde{g}(\varphi(x, t))] \\
& +\tilde{f}(\varphi(x, t))-\tilde{g}(\varphi(x, t)) \\
& +(t \nabla \tilde{f}(\varphi(x, t))+(1-t) \nabla \tilde{g}(\varphi(x, t)), \eta(\varphi(x, t), t))] \\
& \stackrel{(10)}{=}|J \varphi(x, t)|[\operatorname{div} v(\varphi(x, t))+\tilde{f}(\varphi(x, t))-\tilde{g}(\varphi(x, t))] \stackrel{(5)}{=} 0
\end{aligned}
$$

Because of this, we end up with

$$
\begin{aligned}
\frac{1}{g(x)}=\tilde{g}(x) & =|J \varphi(x, 0)| \tilde{g}(\varphi(x, 0)) \\
& =H(x, 0) \\
& =H(x, 1) \\
& =|J \varphi(x, 1)| \tilde{f}(\varphi(x, 1)) \\
& =|J \Phi(x)| \frac{1}{f(\Phi(x))} .
\end{aligned}
$$

As by (5) the normal component of $v$ vanishes for a boundary point $x$, it follows due to formula (6) that $\partial_{\mathfrak{n}} \varphi(x, t)=0 \forall t \in[0,1]$. Therefore, boundary points are moved in tangential direction only.

The existence of such a mapping $\Phi$ is guaranteed by the following theorem from [11]. However, the mapping $\Phi$ is not unique.

Theorem 2.2 (Moser). Let $0<k \in \mathbb{N}, \alpha>0$. Let $\Omega \subset \mathbb{R}^{n}$ be a domain with $\mathcal{C}^{3+k, \alpha}{ }_{-}$ smooth boundary. Suppose $f, g \in \mathcal{C}^{k, \alpha}(\bar{\Omega})$ with $\int_{\Omega} f=\int_{\Omega} g$. Then there exists a $\mathcal{C}^{k+1}$. diffeomorphism $\Phi$ which fulfils

$$
g(x)|J \Phi(x)|=f(\Phi(x)) \quad \forall x \in \Omega
$$

and

$$
\Phi(x)=x \quad \forall x \in \partial \Omega .
$$


Remark 2.3. Note that the derivation of our new deformation method does not depend on the dimension. Although we restrict to the two-dimensional case in this article for the sake of implementational simplicity, our new deformation method is applicable to three-dimensional meshes without any modification.

Remark 2.4. Our new method for grid deformation is a generalisation of Liao's method for static grid deformation. However, our method can be reinterpreted as a special case of a method for time-dependent grid deformation developed by Liao et al. [4, 6], iff the domain itself does not change in time. This dynamic method relies on solving $-\operatorname{div}(v(x, t))=\partial_{t}\left(1 / f_{d}(x, t)\right)$ and then computing the IVPs $\partial_{t} \Phi(x, t)=f_{d}(\Phi(x, t), t) \cdot$ $v(\Phi(x, t), t)$. Setting $1 / f_{d}(x, t):=t \tilde{f}(x)+(1-t) \tilde{g}(x)$, our method is recovered. Both methods can be interpreted [13] as special cases of the GCL method by Cao, Huang and Russell [8].

\section{Convergence analysis}

We consider the fraction

$$
\frac{f(x)}{\operatorname{area}(x)}
$$

as starting point of our convergence analysis. Here, area $(x)$ stands for the interpolated element area distribution of the deformed grid. If $X$ is a vertex of the deformed grid, $\operatorname{area}(X)$ is defined to be the arithmetic mean value of the areas of the elements $X$ belongs to and by the bilinear interpolation of the corresponding node values otherwise. If the desired area distribution is achieved, then the fraction (14) is constant. Iff the function $a(x):=c \cdot \operatorname{area}(x), c \in \mathbb{R}$, fulfils

$$
\int_{\Omega} a(x) d x=\int_{\Omega} f(x) d x
$$

it even holds $f(x) / a(x) \equiv 1$. Now, we define the quality function

$$
q(x):=\frac{f(x)}{a(x)} .
$$

The overall quality of the grid adaptation according to the desired cell size can be measured by the deviation of $q(x)$ from the constant function 1 leading to the quality measures $Q_{0}$ and $Q_{\infty}$ defined by

$$
Q_{0}:=\|q-1\|_{0} \quad \text { and } \quad Q_{\infty}:=\max _{x \in \mathcal{V}}|q(x)-1| .
$$

If we do not distinguish between $Q_{0}$ and $Q_{\infty}$, we omit the subscript.

Remark 3.1. Note that the quality measures defined in equation (16) are consistent with respect to regular refinement, i.e. regular refinement of a deformed grid does not change the quality measures systematically which can be easy shown by standard scaling arguments.

Remark 3.2. Usually, the convergence of a numerical method is defined by comparing the difference of its numerical result and an abstract exact solution. For our deformation 
method, a natural way of defining convergence seems to postulate $\tilde{Q}:=\|\Phi-\tilde{\Phi}\|$ in a certain norm, where $\Phi$ stands for a "reference transformation" obtained by solving all differential equations in the algorithm exactly and $\tilde{\Phi}$ for the approximate transformation computed with numerical methods. However, there are two drawbacks: In contrast to $Q$, $\tilde{Q}$ is hard to compute, as the exact transformation $\Phi$ is of course unknown. More severe, the uniqueness of $\Phi$ in our deformation method is given by the implicit side condition curl $v=0$ in equation (9), which is an artificial condition. Thus there is in fact no unique $\Phi$ to compare with.

When processing our grid deformation algorithm with numerical methods, there are three error sources.

1. The deformation PDE (9) is solved approximately.

2. The IVPs (6) are solved approximately.

3. The interpolation of the discontinuous cell size distribution induces a consistency error.

The consistency error stems from the fact that the actual cell size distribution is discontinuous and has to be interpolated in order to gain area $(x)$. Therefore, even when solving the deformation PDE (9) and all IVPs (6) exactly, we cannot expect one of the quality measures to be zero.

In the following convergence analysis we consider for a given bounded domain $\Omega$ a sequence of triangulations $\left(\mathcal{T}_{i}\right)_{i \in I}$ where $I$ denotes an arbitrary index set. Let us denote the number of vertices in $\mathcal{T}_{i}$ by $N_{i}$. In what follows, we always assume that $\bigcup_{T \in \mathcal{T}_{i}} T=\Omega \forall i \in I$ and $N_{i+1}>N_{i} \forall i \in I$.

The following definitions prepare the convergence analysis of our grid deformation.

Definition 3.3. a) The sequence of triangulations $\left(\mathcal{T}_{i}\right)_{i \in I}$ is said to be edge-length regular, iff

$$
h_{i}:=\max _{e \in \mathcal{E}_{i}}|e|=\mathcal{O}\left(N_{i}^{-1 / 2}\right) \quad \forall i \in I .
$$

b) The sequence of triangulations $\left(\mathcal{T}_{i}\right)_{i \in I}$ is said to be size regular, iff

$$
\exists c, C>0: c h_{i}^{2} \leq m(T) \leq C h_{i}^{2} \quad \forall T \in \mathcal{T}_{i} \forall i \in I .
$$

For the initial grids, we postulate edge-length regularity from now on without explicitly stating this. This property justifies a convergence analysis in powers of $h$. We now define convergence by the decay of our quality measures.

Definition 3.4. For an edge-length regular sequence $\left(\mathcal{T}_{i}\right)_{i \in I}$, a sequence of grid deformations is said to converge, iff $Q \rightarrow 0$ for $h \rightarrow 0$. Here, $Q$ stands for either $Q_{0}$ or $Q_{\infty}$

Definition 3.5. An edge-length regular sequence of triangulations $\left(\mathcal{T}_{i}\right)_{i \in I}$ fulfils the similarity condition, iff there is a function $g$ with $0<g_{\min } \leq g(x) \leq g_{\max }<\infty$ and there are positive constants $c_{s}, C_{s}$ with

$$
\frac{1}{h_{i}^{2}} c_{i} m(T)=g(x)+\mathcal{O}\left(h_{i}\right) \quad \forall x \in T \quad \forall T \in \mathcal{T}_{i} \forall i \in I, \quad c_{s} \leq c_{i} \leq C_{s} .
$$

Here, $c_{i}$ is a spatially fixed constant. 
If a sequence of grids fulfils the similarity conditions, all grids feature a similar distribution of element sizes up to a spatially fixed constant. All three conditions on the mesh defined here are naturally satisfied for a sequence of grids created by successive regular refinement of an arbitrary coarse grid. Notice that these requirements affect the asymptotic behaviour only and permit large spatial variations of both element size and shape.

For edge-length regular sequences of triangulations, the similarity condition implies size regularity.

Lemma 3.6. Let the sequence of triangulations $\left(\mathcal{T}_{i}\right)_{i \in I}$ fulfil the similarity condition. Then, it is size regular.

Proof. Consider an arbitrary element $T \in \mathcal{T}_{i}$. Then,

$$
m(T)=\frac{h_{i}^{2}}{c_{i}} g(x)+\underbrace{\mathcal{O}\left(h_{i}^{3}\right)}_{\leq c h_{i}^{3}} \leq\left(\frac{g_{\max }}{c_{s}}+c\right) h_{i}^{2}
$$

for $h_{i}<1$. On the other hand,

$$
m(T)=\underbrace{\frac{h_{i}^{2}}{c_{i}} g(x)}_{>0}+\underbrace{\mathcal{O}\left(h_{i}^{3}\right)}_{\geq-c h_{i}^{3}} \geq\left(\frac{g_{\min }}{C_{s}}-c h_{i}\right) h_{i}^{2} \geq \frac{g_{\min }}{2 C_{s}} h_{i}^{2}
$$

for $h_{i}<g_{\min } /\left(c C_{s}\right)$.

To formulate the following results, we need some notations. For an arbitrary grid point $x$, we denote by $X$ its image in the deformed grid obtained with both PDE (9) and all IVPs (6) solved exactly. Let us denote by $X_{h}$ the image of the same vertex $x$, but computed by solving exactly the disturbed initial value problem, where the exact vector field $v$ is replaced by $v_{h}$ in the right hand side. Furthermore, $\tilde{X}$ is the corresponding point obtained by computing both the PDE and the IVP numerically. The following two lemmas demonstrate that size and shape regularity are preserved under grid deformation, if our deformation algorithm is carried out solving all differential equations exactly. As an abbreviation we call these grids "exactly deformed", and "numerically deformed" if the differential equations have been solved by numerical methods.

Lemma 3.7. Let $\left(\mathcal{T}_{i}\right)_{i \in I}$ be a sequence of grids with grid size $h_{i} \rightarrow 0$ which fulfils the similarity condition (18). Then the sequence $\left(\mathcal{T}_{i}{ }^{d}\right)_{i \in I}$ of exactly deformed meshes is edgelength regular.

Proof. Let us denote by $X$ and $Y$ the images of the vertices $x$ and $y$ obtained by exact computation. These vertices are computed by solving the initial value problems

$$
\begin{array}{ll}
\partial_{t} \varphi^{x}(t)=\eta\left(\varphi^{x}(t), t\right), & \varphi^{x}(t=0)=x \\
\partial_{t} \varphi^{y}(t)=\eta\left(\varphi^{y}(t), t\right), & \varphi^{y}(t=0)=y
\end{array}
$$

with the right hand side $\eta$ defined in formula (6). According to Gronwall's lemma (compare $[16$, p. 57$])$, it holds

$$
\left\|\varphi^{x}(t)-\varphi^{y}(t)\right\| \leq e^{L t}(\underbrace{\left\|\varphi^{x}(0)-\varphi^{y}(0)\right\|}_{=\|x-y\|}) .
$$


Here, the Lipschitz constant $L$ of the ODE right hand side is defined by

$$
\left\|\eta(t, x)-\eta\left(t, x^{\prime}\right)\right\| \leq L\left\|x-x^{\prime}\right\| \forall x, x^{\prime} \in \Omega \forall t \in[0,1] .
$$

Thus, we have

$$
\begin{aligned}
L & \leq \sup _{t \in[0,1]} \sup _{x, x^{\prime} \in \Omega, x \neq x^{\prime}}\left\|\frac{v(x)}{s f(x)+(1-s) g(x)}-\frac{v\left(x^{\prime}\right)}{s f\left(x^{\prime}\right)+(1-s) g\left(x^{\prime}\right)}\right\|\left\|x-x^{\prime}\right\|^{-1} \\
& =\mathcal{O}\left(h^{0}\right) .
\end{aligned}
$$

From this, we deduce for $t=1$

$$
\left\|\varphi^{x}(1)-\varphi^{y}(1)\right\|=\|X-Y\| \leq e^{L}\|x-y\| \leq e^{L} \operatorname{ch} .
$$

Lemma 3.8. Let $f>\varepsilon>0$ be a strictly positive monitor function, $f \in \mathcal{C}^{1}(\bar{\Omega})$ and $\left(\mathcal{T}_{i}\right)_{i \in I}$ be a sequence of grids which fulfils the similarity condition (18). Then, the sequence $\left(\mathcal{T}_{i}^{d}\right)_{i \in I}$ of exactly deformed grids is size regular.

Proof. Because of $f \in \mathcal{C}^{1}(\bar{\Omega}), \exists f_{\max }:=\max _{x \in \Omega}|f(x)|<\infty$. By definition 3.5, it holds $g_{\min } \leq g \leq g_{\max }$. Thus,

$$
\frac{m(\Phi(T))}{m(T)} \leq 2 \frac{f_{\max }}{g_{\min }}=\mathcal{O}\left(h^{0}\right)
$$

and, vice versa,

$$
\frac{m(T)}{m(\Phi(T))} \leq 2 \frac{g_{\max }}{\varepsilon}=\mathcal{O}\left(h^{0}\right)
$$

for $h<h_{0}$. Therefore, $m(T)$ and $m(\Phi(T))$ have the same convergence order. As the sequence of initial grids is size regular due to lemma 3.6, the assertion follows immediately.

Now we are able to formulate and prove our central convergence theorem for grid deformation.

Theorem 3.9. Let $\left(\mathcal{T}_{i}\right)_{i \in I}$ be a sequence of grids with grid size $h_{i} \rightarrow 0$ which fulfils condition (18) and let us denote the sequence of numerically deformed grids by $\left(\tilde{\mathcal{T}}_{i}\right)_{i \in I}$. For the monitor function $f$, it may hold $0<\varepsilon<f \in \mathcal{C}^{1}(\bar{\Omega})$. Let us assume that for the approximate solution of the deformation vector field $v_{h}$, the equation $\left\|v-v_{h}\right\|_{\infty}=$ $\mathcal{O}\left(h^{1+\delta}\right), \delta>0$ is valid. Let $\left\|X_{h}-\tilde{X}\right\|=\mathcal{O}\left(h^{1+\delta}\right)$ be true for any vertex. Then,

a) the sequence of numerically deformed meshes $\left(\tilde{\mathcal{T}}_{i}\right)_{i \in I}$ is edge-length regular,

b) $\left(\tilde{\mathcal{T}}_{i}\right)_{i \in I}$ is size regular according to condition (17),

c) the sequence of deformations converges, moreover, $\exists c>0$ independent of $h$, such that

$$
Q_{0} \leq c h^{\min \{1, \delta\}}, \quad Q_{\infty} \leq c h^{\min \{1, \delta\}} .
$$




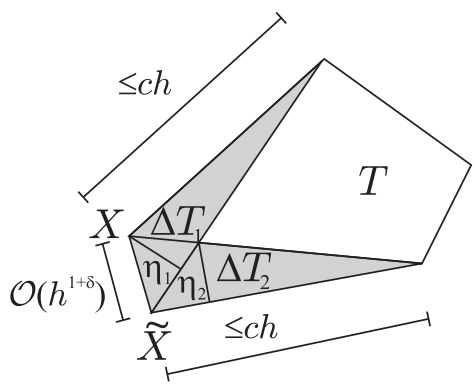

Figure 3.1: Influence of an disturbed vertex on the element area

Proof. Let us denote the area distribution of the numerically deformed grid $T$ by $\tilde{a}(x)$. The quantities $X_{h}$ and $X$ are computed as the solution of the initial value problems

$$
\begin{aligned}
\partial_{t} \varphi(t) & =\eta(\varphi(t), t), \quad \varphi(0)=x, \\
\partial_{t} \varphi_{h}(t) & =\eta_{h}\left(\varphi_{h}(t), t\right), \quad \varphi_{h}(0)=x
\end{aligned}
$$

in $t=1$. As a consequence of Gronwall's lemma, it holds (compare [16, p. 57]) that

$$
\begin{aligned}
\left\|X-X_{h}\right\| & \leq e^{L} \int_{0}^{1} \sup _{x \in \Omega}\left\|\eta(x, s)-\eta_{h}(x, s)\right\| d s \\
& \leq e^{L} \int_{0}^{1} \sup _{x \in \Omega}\left\|\frac{v(x)-v_{h}(x)}{s f(x)+(1-s) g(x)}\right\| d s \\
& \leq e^{L} \underbrace{\sup _{x \in \Omega}\left\|v(x)-v_{h}(x)\right\|}_{=\mathcal{O}\left(h^{1+\delta}\right)} \underbrace{\sup _{t \in[0,1]} \sup _{x \in \Omega}\left\|(t f(x)+(1-t) g(x))^{-1}\right\|}_{=\mathcal{O}\left(h^{0}\right)} \\
& =e^{L} \mathcal{O}\left(h^{1+\delta}\right) .
\end{aligned}
$$

Here, the Lipschitz constant $L$ is given by the condition (19) and fulfils equation (20) like in the proof of lemma 3.7. The combination of this upper bound with formula (22) yields

$$
\left\|X-X_{h}\right\|=\mathcal{O}\left(h^{1+\delta}\right) .
$$

for all grid vertices $X$. Using the triangle inequality, it follows

$$
\|X-\tilde{X}\| \leq \underbrace{\left\|X-X_{h}\right\|}_{(23): \mathcal{O}\left(h^{1+\delta}\right)}+\underbrace{\left\|X_{h}-\tilde{X}\right\|}_{=\mathcal{O}\left(h^{1+\delta}\right)}=\mathcal{O}\left(h^{1+\delta}\right) .
$$

Note that equation (24) holds without any assumptions on the initial and deformed mesh. Let $\tilde{e}$ be an arbitrary edge on the numerically deformed grid with endpoints $\tilde{X}$ and $\tilde{Y}$. Then, due to the triangle inequality and lemma 3.7 , it follows

$$
\|\tilde{X}-\tilde{Y}\| \leq \underbrace{\|X-\tilde{X}\|}_{(24): \leq c h^{1+\delta}}+\underbrace{\|X-Y\|}_{\leq c h}+\underbrace{\|Y-\tilde{Y}\|}_{(24): \leq c h^{1+\delta}} \leq c h,
$$

and thus the edge-length regularity of the deformed grid. Let us consider a single element $T$ in the numerically computed deformed grid $\tilde{X}$ is a vertex of. Let us denote by $X$ the 
exactly computed counterpart of $\tilde{X}$. Then, the area difference $\Delta T$ due to the disturbed vertex $\tilde{X}$ can be bounded by $\Delta T \leq \Delta T_{1}+\Delta T_{2}$ (see figure 3.1). These quantities are the areas of triangles which basis length is bounded by ch due to the shape regularity. Their heights $\eta_{1}$ and $\eta_{2}$ are not larger than $\|X-\tilde{X}\|$ and therefore (at most) $\mathcal{O}\left(h^{1+\delta}\right)$. Because of this, $\Delta T$ due to the disturbed vertex $\tilde{X}$ can be bounded by $c_{d} h^{2+\delta}$. This holds for the other vertices of $T$ as well, so that we can conclude

$$
m(\Phi(T))=m(\tilde{\Phi}(T))+\mathcal{O}\left(h^{2+\delta}\right) .
$$

From this, we get using lemma 3.8

$$
m(\tilde{\Phi}(T)) \leq \underbrace{m(\Phi(T))}_{\leq C h^{2}}+c_{d} h^{2+\delta} \leq 2 C h^{2}
$$

and vice versa

$$
m(\tilde{\Phi}(T)) \geq \underbrace{m(\Phi(T))}_{\geq c h^{2}}-c_{d} h^{2+\delta} \geq \frac{1}{2} c h^{2}
$$

for $h<h_{0}$. This proves assertion b).

By construction, $\tilde{a}(x)=\frac{c}{h^{2}} m(\tilde{\Phi}(T))+\mathcal{O}(h)$ holds. By a Taylor expansion of the monitor function $f$, we obtain

$$
\begin{aligned}
\frac{f(\tilde{X})}{\tilde{a}(\tilde{X})} & =\frac{f(X)+\nabla f(\chi) \cdot(X-\tilde{X})}{\frac{c}{h^{2}} m(\tilde{\Phi}(T))+\mathcal{O}(h)} \\
& =\frac{f(X)}{\frac{c}{h^{2}} m(\tilde{\Phi}(T))+\mathcal{O}(h)}+\underbrace{\frac{\overbrace{\nabla f(\chi) \cdot(X-\tilde{X})}^{=\mathcal{O}\left(h^{1+\delta}\right)}}{\frac{c}{h^{2} m(\tilde{\Phi}(T))+\mathcal{O}(h)}}}_{=\mathcal{O}\left(h^{0}\right)} .
\end{aligned}
$$

Inserting equation (25), we obtain

$$
\frac{f(\tilde{X})}{\tilde{a}(\tilde{X})}=\frac{f(X)}{\frac{c}{h^{2}} m(\Phi(T))+\mathcal{O}\left(h^{\min \{1, \delta\}}\right)}+\mathcal{O}\left(h^{1+\delta}\right)
$$

Now, we exploit that due to the edge-length regularity of the deformed mesh, the length of any element edge is bounded from above by $c h$. Let us denote the center of the undeformed element by $x_{c}$. Then we get by another Taylor expansion

$$
\begin{aligned}
\frac{f(\tilde{X})}{\tilde{a}(\tilde{X})} & =\frac{f\left(\Phi\left(x_{c}\right)\right)}{\frac{c}{h^{2}} m(\Phi(T))+\mathcal{O}\left(h^{\min \{1, \delta\}}\right)}+\frac{\overbrace{\nabla f(\nu) \cdot\left(\Phi\left(x_{c}\right)-X\right)}^{=\mathcal{O}(h)}}{\underbrace{\frac{c}{h^{2} m(\Phi(T))+\mathcal{O}\left(h^{\delta}\right)}}_{=\mathcal{O}\left(h^{0}\right)}}+\mathcal{O}\left(h^{1+\delta}\right) \\
& =\frac{f\left(\Phi\left(x_{c}\right)\right)}{f\left(\Phi\left(x_{c}\right)\right)}+\mathcal{O}\left(h^{\min \{1, \delta\}}\right)+\mathcal{O}(h) \\
& =1+\mathcal{O}\left(h^{\min \{1, \delta\}}\right) .
\end{aligned}
$$


As this relation holds for all grid vertices, it follows immediately the assertion $Q_{\infty}=$ $\mathcal{O}\left(h^{\min \{1, \delta\}}\right)$. Let now $Y$ be an arbitrary grid point in the interior of the element $T$ with vertices $V_{1}, \ldots V_{4}$ in the numerically deformed grid. By $\tilde{X}$, we denote one of the four vertices of $T$ where the area function $\tilde{a}$ is minimal: $\tilde{a}(\tilde{X})=\min _{i=1}^{4}\left\{\tilde{a}\left(V_{i}\right)\right\}$. As the computed grid is edge-length regular and size regular according to condition (17) and due to the normalisation condition (15)), we know that $\tilde{a}(\tilde{X})=\mathcal{O}\left(h^{0}\right)$. Moreover, as $\tilde{a}$ is a bilinear function on $T$, we have $\tilde{a}(Y) \geq \tilde{a}(\tilde{X}) \forall Y \in T$. Thus, we gain by a Taylor expansion

$$
\frac{f(Y)}{\tilde{a}(Y)} \leq \frac{f(Y)}{\tilde{a}(\tilde{X})}=\frac{f(\tilde{X})}{\tilde{a}(\tilde{X})}+\frac{\overbrace{\nabla f(\mu) \cdot(\tilde{X}-Y)}^{\leq c h}}{\tilde{a}(\tilde{X})} \leq \frac{f(\tilde{X})}{\tilde{a}(\tilde{X})}+c h
$$

for sufficiently small $h$. This leads to the assertion $Q_{0} \leq c h^{\min \{1, \delta\}}$.

\section{Numerical Realisation and Analysis}

This section is devoted to the numerical implementation of the four steps described above. Although the construction of the mapping $\Phi$ can be performed in any dimension, we restrict ourselves to the two-dimensional case. For first investigations of the threedimensional case, we refer to the master thesis of Panduranga [22] and the diploma thesis of Miemczyk [21]. To prove the existence of a smooth diffeomorphism $\Phi$, Moser uses the smoothness conditions of $f, g$ and the domain $\Omega$ stated above. In practical computations, we relax these conditions. In the examples presented in this article, the functions $f$ and $g$ are strictly positive and continuous, the domain $\Omega$ may have a Lipschitz boundary.

The first step in constructing $\Phi$ is to obtain the functions $f$ and $g$. In our computations, the monitor function $f$ is defined to be the bilinear interpolant of an analytically given function. To compute $g$, we first determine the element size in a grid point, which is set as the arithmetic mean of the area of the elements surrounding the grid point. Then, we define $g$ as the bilinear interpolant of these node values.

To maintain a high degree of flexibility with respect to the underlying mesh, we compute in the second step of our algorithm the Poisson problem (9) using its discrete weak formulation

$$
\left(\nabla w_{h}, \nabla \varphi_{h}\right)=\left(\tilde{f}-\tilde{g}, \varphi_{h}\right) \quad \forall \varphi_{h} \in \mathcal{Q}_{1}(\mathbb{T})
$$

by the finite element method on the given mesh $\mathbb{T}$. Here $\mathcal{Q}_{1}$ denotes the function space created by continuous and elementwise bilinear functions on $\mathbb{T}$. In the following, every FEM calculation is performed with bilinear conforming finite elements.

The solution of the corresponding algebraic systems however requires special care, as the solution of the Neumann problem (9) is unique up to an additive constant only. To solve (9), we use a modified multigrid method in which after every iteration the side condition $\int_{\Omega} w_{h} d x=0$ is imposed by adding a suitable constant [13].

Afterwards, the vector field $v$ is approximated by the recovered gradient $v_{h}$ of the finite element solution $w_{h}$. For the reconstruction of the gradient we employ standard averaging techniques.

In the next step, we approximate the initial value problem (6) by replacing $v$ by its discrete counterpart $v_{h}$. This leads to the initial value problem

$$
\frac{\partial \varphi(x, t)}{\partial t}=\eta_{h}(\varphi(x, t), t), \quad 0 \leq t \leq 1, \quad \varphi(x, 0)=x
$$


with

$$
\eta_{h}(y, s):=\frac{v_{h}(y)}{s \tilde{f}(y)+(1-s) \tilde{g}(y)}, \quad y \in \Omega, s \in[0,1] .
$$

Note that this ODE system decouples into 1D-ODEs for every coordinate, which can be solved by standard ODE methods.

As IVP solver, we apply the explicit Euler's method (EE) and several Runge-Kutta type methods: Heun's method (HEUN), the classical Runge method of third order (RK3) and the standard Runge-Kutta method of fourth order (RK4) These convergence orders, however, can be experienced only for sufficiently smooth solutions and thus sufficiently smooth right hand sides. The right hand side we consider is continuous only and therefore lacks the required smoothness. Thus, we can not expect to experience full convergence of the high order methods. Because of this, we additionally investigate the Runge-KuttaFehlberg method RKF2B (see [16, p. 167]). This scheme is a three-stage method, but of second order only. Instead of aiming at the highest possible convergence order (three), this method is designed to achieve a particularly small error constant. Furthermore, we employ Adams-Bashforth methods of order two and three (AB2, AB3) as representatives of linear multi-step methods. The starting values are obtained by Heun's method. For all IVP solvers mentioned, we refer to Hairer, Norsett and Wanner [16].

Remark 4.1. In the proposed method for grid deformation, every evaluation of the right hand inside formula (6) in the ODE solve requires searching through the grid: To evaluate a finite element function in a given point in real coordinates, the element this point belongs to has to be known. In practical implementations, it is crucial for computational efficiency to employ fast and sophisticated searching methods. We propose raytracing and distance search (for details, we refer to Grajewski [13]).

All numerical tests in this article refer to the following test problem. Figure 4.1 displays a resulting grid with 1,024 elements.

Test Problem 4.2. We consider the unit square $\Omega=[0,1]^{2}$ triangulated by a tensor product mesh. As monitor function, we choose

$$
f(x)=\min \left\{1, \max \left\{\frac{|d-0.25|}{0.25}, \epsilon\right\}\right\}, \quad d:=\sqrt{\left(x_{1}-0.5\right)^{2}+\left(x_{2}-0.5\right)^{2}} .
$$

The parameter $\epsilon$ is set to 0.1. This setting implies that on the deformed grid the largest cell has 10 times the area of the smallest one.

The right hand side of the ODE in the deformation method is formed by a piecewise rational function which is continuous but not differentiable in a classical sense. Therefore, the right hand side of the ODE lacks the regularity needed for higher order IVP solvers to establish full convergence. Now, we investigate if and to what extend the convergence order of the high order methods suffers decay. We compute test problem 4.2 with fixed step size $10^{-6}$ employing the classical RK3 scheme on a tensor product grid. The deviation $\rho$ from this reference grid induced by the IVP solver is measured by taking the $l^{\infty}$-norm of the vector containing the pointwise deviation defined by $\left\|x_{\text {ref }}-x\right\|$ where $x_{\text {ref }}$ stands for the position of the corresponding point on the reference grid. Note that our reference grid is not the grid obtained by applying the deformation algorithm without any approximation, because the deformation vector field is computed as recovered 


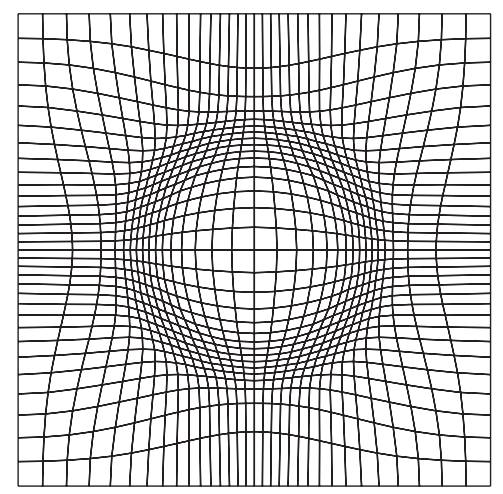

Figure 4.1: Resulting grid for test problem 4.2, 1,024 elements

gradient of an FEM solution as before. However, in the deformation algorithm, the error stemming from the PDE approximation is fully decoupled from the error coming from the IVP approximation. This allows to investigate these errors separately and thus justifies the numerical tests presented here. Figures 4.2 and 4.3 which visualize $\rho$ vs. the number of evaluations of the ODE right hand side indicate that all high order IVP methods are of second order only in the example considered. However, the high order methods (AB3, RK3, RK4) perform best in the sense that given a fixed number of right hand side evaluations, the absolute approximation error related to these methods is smaller than the one of low order methods. The RKF2B method seems to be comparable to the high order Runge-Kutta methods in this numerical test. Note that when considering $\rho$, the advantages of the Runge-Kutta methods in favour of the linear multi-step methods are far less pronounced than the quality measures indicated before. Remarkably, the RungeKutta method RK4 produces less accurate results per evaluation of the right hand side than the (actually lower order) RK3-method for this example.

We now return to investigations on the overall convergence behaviour. For ease of implementation, we will replace from now on in the computations of the quality measures the analytical monitor function $f$ by its bilinear interpolant $f_{h}$ on the deformed grid which is scaled then in order to fulfil equation (15). This leads to the scaled interpolant $\hat{f}$. Note that the integrals of $f$ and $f_{h}$ do not need to coincide which requires the additional scaling. The following lemma justifies our approach as it is shown that the additional error due to this replacement is of higher order.

Lemma 4.3. Let the assumptions in theorem 3.9 be fulfilled. Let $Q$ denote the quality measures computed with the analytical monitor function $f$ and the same measure $\hat{Q}$ obtained using $\hat{f}$ instead. Here, $Q$ stands for either $Q_{0}$ or $Q_{\infty}$. Then,

$$
Q=\hat{Q}+\mathcal{O}\left(h^{2}\right) \text {. }
$$

Proof. Because of $f \in \mathcal{C}^{1}(\Omega)$, we know

$$
\max _{x \in \Omega}\left|f(x)-f_{h}(x)\right| \leq C h^{2} .
$$

Let us denote the factor the function $f_{h}$ is scaled with by $\omega$. Then,

$$
\omega \int_{\Omega} f_{h}(x) d x=|\Omega|=\int_{\Omega} f(x) d x
$$




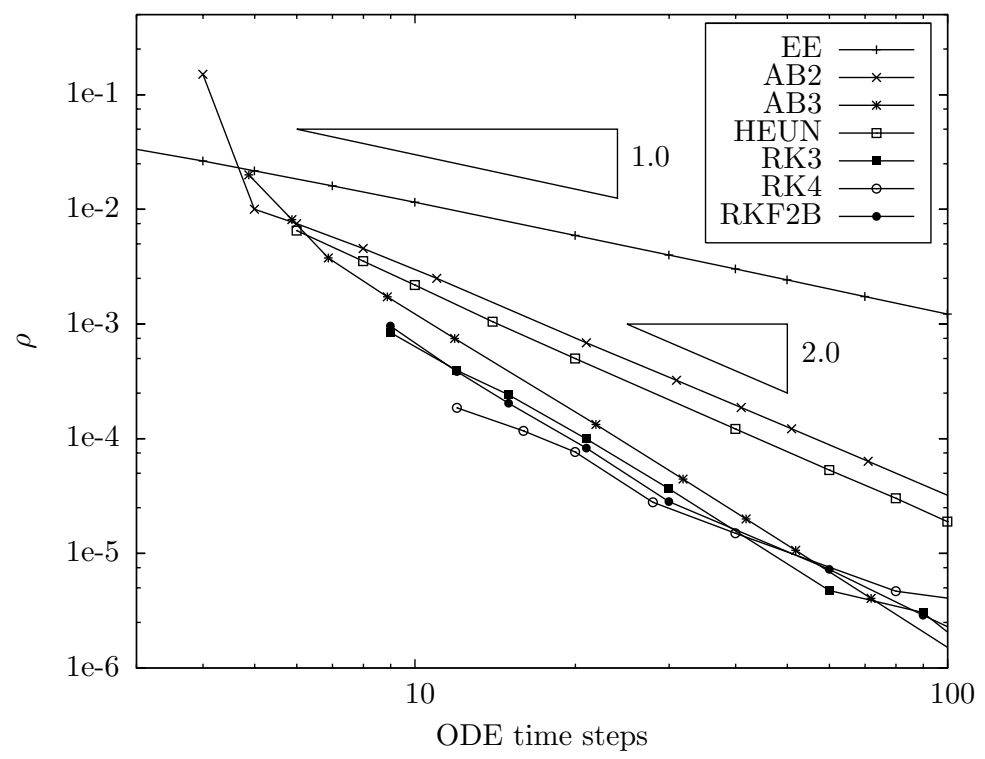

Figure 4.2: Deviation $\rho$ and order of convergence of several ODE methods in the case of test problem 4.2, 4,096 elements

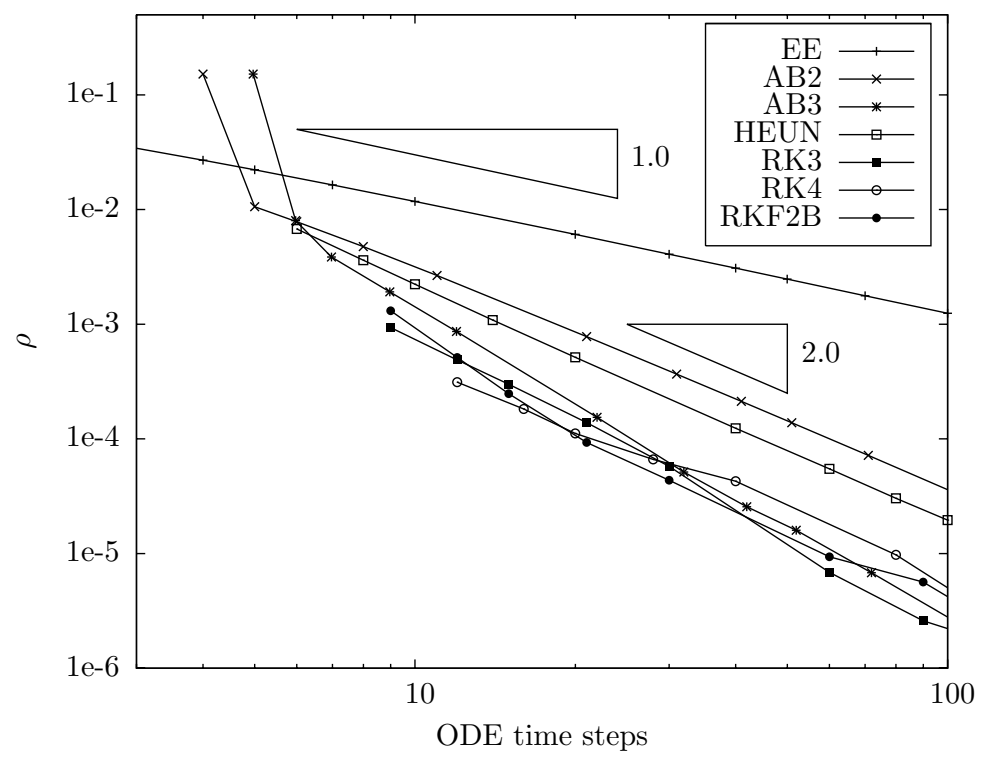

Figure 4.3: Deviation $\rho$ and order of convergence of several ODE methods in the case of test problem 4.2, 65,536 elements 
and thus

$$
\omega=\frac{\int_{\Omega}\left(f-f_{h}\right)(x) d x}{\int_{\Omega} f(x) d x}+1 \leq \frac{C|\Omega|}{\int_{\Omega} f_{h}(x) d x} h^{2}+1 .
$$

Vice versa

$$
\frac{1}{\omega}=\frac{\int_{\Omega}\left(f_{h}-f\right)(x) d x}{\int_{\Omega} f(x) d x}+\frac{\int_{\Omega} f(x) d x}{\int_{\Omega} f(x) d x} \leq C h^{2}+1 .
$$

Therefore, $\exists 0<c_{1}, C_{1}$ such that $1-c_{1} h^{2} \leq \omega \leq 1+C_{1} h^{2}$. Thus, we conclude $\omega-1=$ $\mathcal{O}\left(h^{2}\right)$ and because of this

$$
\begin{aligned}
\max _{x \in \Omega}|f(x)-\hat{f}(x)| & =\max _{x \in \Omega}\left|f-\left(1+\mathcal{O}\left(h^{2}\right)\right) f_{h}(x)\right| \\
& \leq \max _{x \in \Omega}\left|f(x)-f_{h}(x)\right|+\mathcal{O}\left(h^{2}\right) \max _{x \in \Omega}\left|f_{h}(x)\right| .
\end{aligned}
$$

Consequently, we end up with

$$
Q=\left\|\frac{f}{g}-1\right\| \leq\left\|\frac{(f-\hat{f})}{g}\right\|+\left\|\frac{\hat{f}}{g}-1\right\|=\hat{Q}+\mathcal{O}\left(h^{2}\right) .
$$

With a similar argument, one can show that the replacement of $f$ by $\hat{f}$ in the grid deformation itself does not alter the deformation method substantially as well. As thus the change from $f$ to $\hat{f}$ does not have any significant influence on the convergence and the quality measures, we from now on implicitly assume this replacement for all numerical examples and experiments, writing $f$ instead of $\hat{f}$ then.

Remark 4.4. In practical computations, the computation of $Q_{0}$ requires numerical integration which introduces an additional error. Note that even on tensor product meshes the quotient of the bilinear functions $f$ and area is not bilinear but rational. Because of this, it is impossible with standard quadrature rules to avoid any quadrature error unlike in the case of bilinear functions. In our examples, we employ the (third order) tensor product Simpson rule for computing $Q_{0}$. Thus, the quadrature error can be asymptotically neglected. To compute $Q_{\infty}$, we use the quadrature points of the Simpson rule as sample points.

Corollary 4.5. Let us assume that our numerical method for computing the deformation IVPs is of second order as indicated by the numerical tests presented above. Let all initial grids be size- and shape-regular and fulfil the similarity condition (18) For the monitor function $f$ and the sequence of deformed grids, the assumptions of theorem 3.9 may hold. Furthermore, let us choose the step size $\Delta t$ of the deformation IVPs as $\Delta t=\mathcal{O}(h)$. Then, if $\left\|v-v_{h}\right\|_{\infty}=\mathcal{O}\left(h^{2}\right)$ and if the IVP method is of second order at least,

$$
Q_{0} \leq c h, \quad Q_{\infty} \leq c h .
$$

Proof. Due to the choice of IVP time steps, we have (in the notation of theorem 3.9), $\left\|X_{h}-\tilde{X}\right\|=\mathcal{O}\left(h^{2}\right)$ for all grid vertices. Thus, the preliminaries of theorem 3.9 are fulfilled with $\delta=1$.

Remark 4.6. The rather strong assumption $\left\|v-v_{h}\right\|_{\infty}=\mathcal{O}\left(h^{2}\right)$ is justified in the situation of our test problem due to the high regularity of the mesh and the solution of the Poisson equation. For superconvergence results in the maximum norm, we refer to Blum [3] and the references cited therein. 


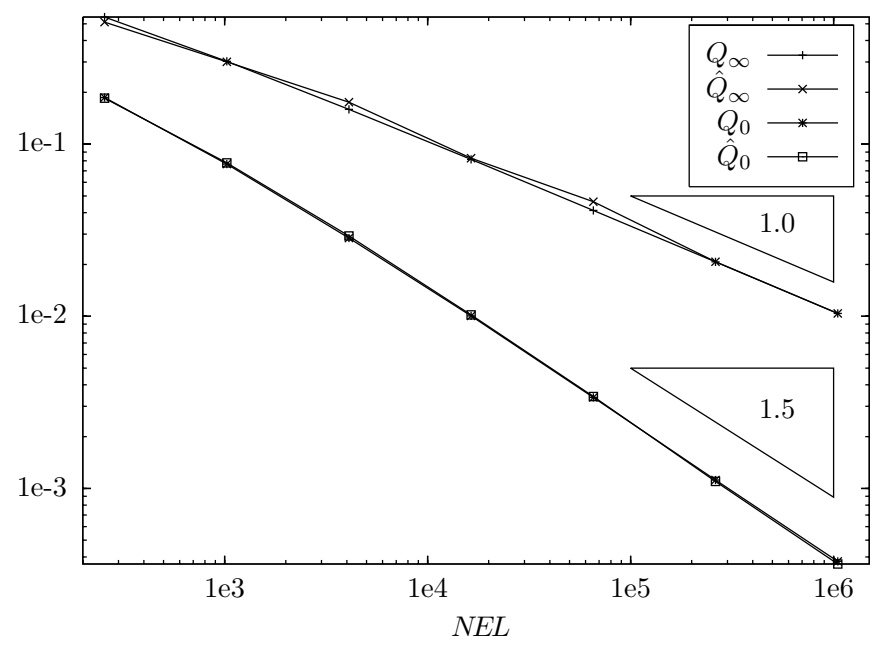

Figure 4.4: Quality measure $Q_{0}$ and $Q_{\infty}$ computed with the true monitor function $f$ and quality measures $\hat{Q}_{0}, \hat{Q}_{\infty}$ using the bilinear interpolant $\hat{f}$ vs. number of elements

To verify our convergence results, we compute test problem 4.2 using standard averaging techniques to obtain the deformation vector field $v_{h}$ and compute the deformation IVPs with RK3. The sequence of initial grids consists of successively refined tensor product grids. Note that in this test setting, there is no consistency error for the representation of the area distribution of the initial mesh in contrast to the general case. On the coarsest grid with $N E L=256$, we perform 3 RK3-steps, on the grid with $N E L=$ 1,024 , we employ 5. From this level of refinement on, the number of IVP steps doubles per refinement. In figure 4.4, we display the quality measures $Q_{0}$ and $Q_{\infty}$ and their approximate counterparts $\hat{Q}_{0}$ and $\hat{Q}_{\infty}$ depending on the number of elements $N E L$. Clearly, we observe $Q \leq c h$ for all quality measures as expected. Note that the monitor function in our test problem is Lipschitz-continuous, but not differentiable, and thus the assumptions of our convergence theorem are not completely fulfilled. For $Q_{0}$, we even experience $Q_{0}=\mathcal{O}\left(h^{3 / 2}\right)$. The reason for this superconvergence phenomenon is subject of ongoing research. Moreover, figure 4.5 demonstrates that the sequence of deformed grids is edge-length regular as stated in our theorem. Additionally, due to the order of $h_{\min }:=\min _{e \in \mathcal{E}}|e|$, we can conclude that $|e| \geq c h \forall e \in \mathcal{E}$.

\section{$5 \quad$ Applications and outlook}

In this article, the emphasis is put on the derivation and mathematical analysis of our the presented grid deformation method. However, the algorithm presented and analysed in this article is only the basic version of a class of grid deformation methods which feature outstanding accuracy, speed and robustness. A series of forthcoming papers [14, 15] focuses on these algorithms, their efficient implementation, their asymptotic complexity as well as their embedding in $r$-and $r h$-adaptive algorithms driven by a posteriori error estimation. Variants of this grid deformation method have been sucessfully applied to many practical problems. We now show in brief two of these applications.

Turek and Wan [23] investigated the numerical simulation of rigid particulate flows 


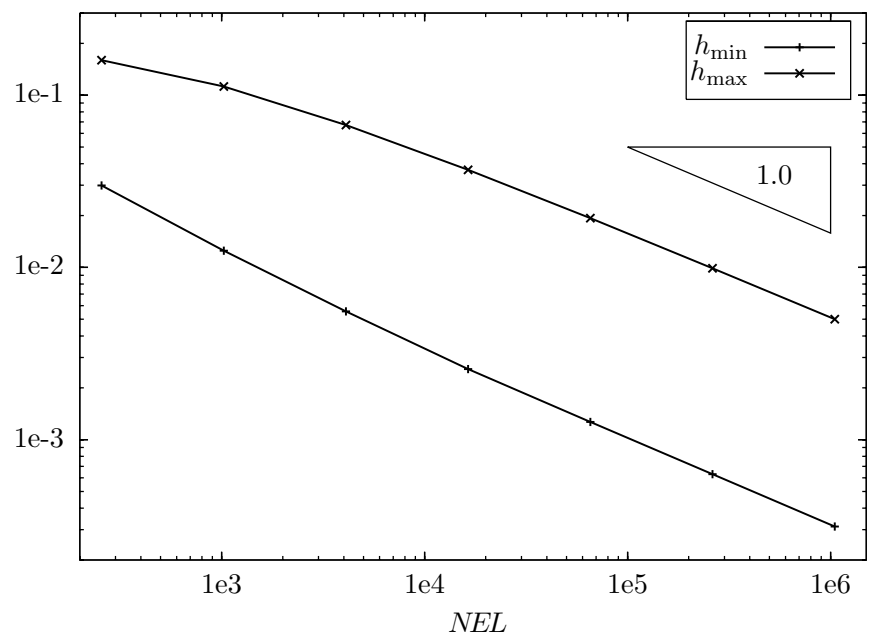

Figure 4.5: Minimal and mximal edge length $h_{\min }$ and $h_{\max }$ vs. number of elements

using a variant of the grid deformation method presented in this article in combination with fictitious boundary method (FBM). The flow field was computed by a special ALE formulation of the Navier-Stokes equations to compensate the mesh deformation in time. The equation was discretised by nonconforming Finite Elements and the resulting linear subproblems were solved with multigrid techniques. Solid particles could move freely through the computational mesh which was adapted by grid deformation using the distance to the particles as monitor function. Numerical results show the benefit of grid deformation in particulate flows with many moving rigid particles. Among many examples, Turek and Wan considered the sedimentation of 120 balls of identical size and shape falling down in a closed channel. Grid deformation was used in this example to concentrate the mesh around the falling balls. We present a sequence of meshes created during this simulation in figure 5.1.

In his diploma thesis [21], Miemczyk applied the grid deformation method presented in this article in three dimensions for the computation of the flow around objects in a channel using FBM. The flow field was governed by the nonstationary incompresible Navier-Stokes equations. The monitor function in his examples was chosen according to the distance to the object. Thus, the grid was concentrated around its contours which helped to reduce the boundary approximation error on the object and therefore improved the accuracy of the computation. In figure 5.2, we show a grid adapted by our deformation method for simulating the flow around a yacht.

\section{Acknowledgements}

We want to thank Susanne Kilian and Reinhard Zwirnmann for a first implementation of Liao's dynamic method which inspired us to further research on this kind of grid deformation method. Furthermore, we thank Matthias Miemczyk for providing the image in figure 5.2. This work is partially supported by the German Research Association (DFG) through the collaborative research center SFB 708 and through the grant TU 102/24-1 (SPP 1253). 

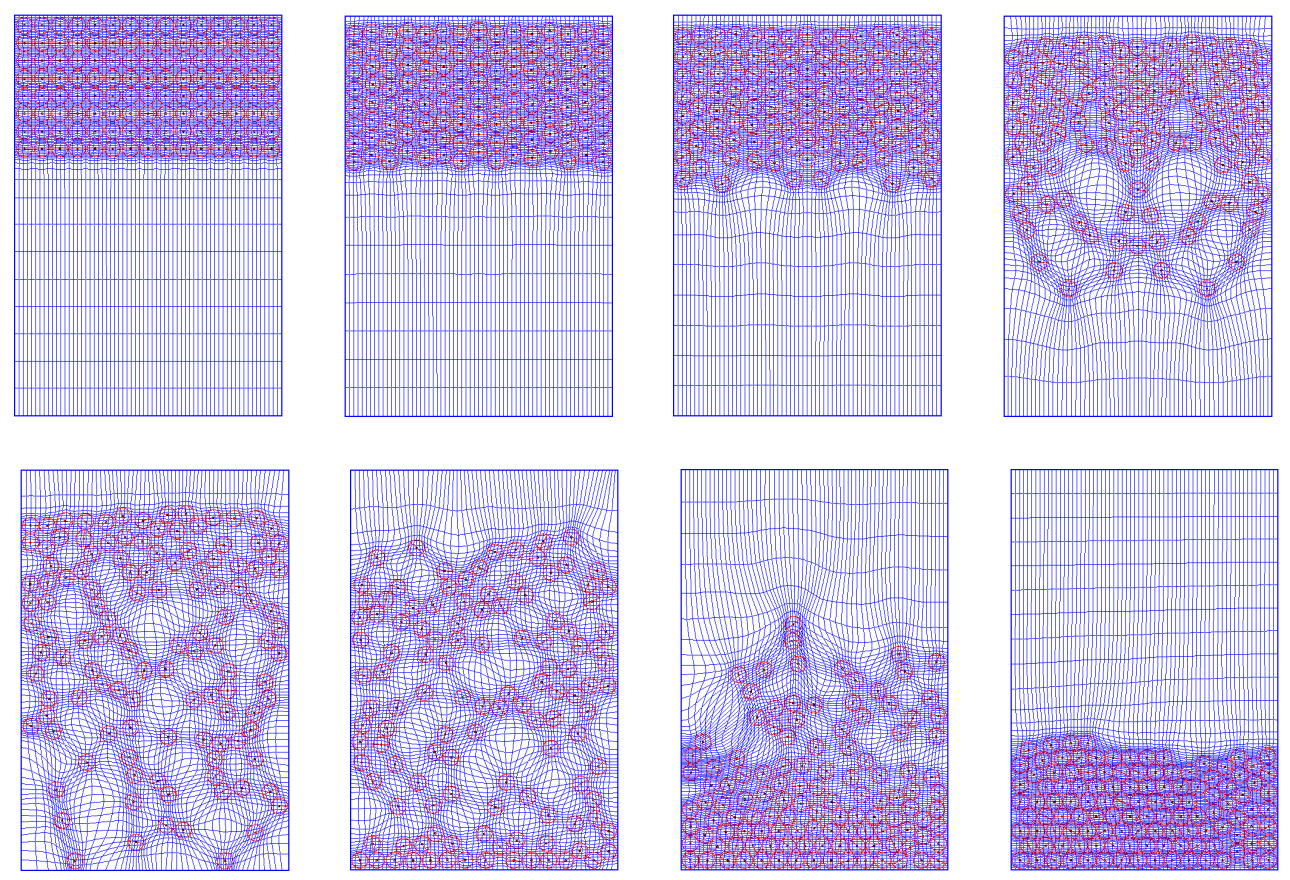

Figure 5.1: Sequence of adapted grids for the simulation of the sedimentation of 120 balls (taken from Wan and Turek [23])

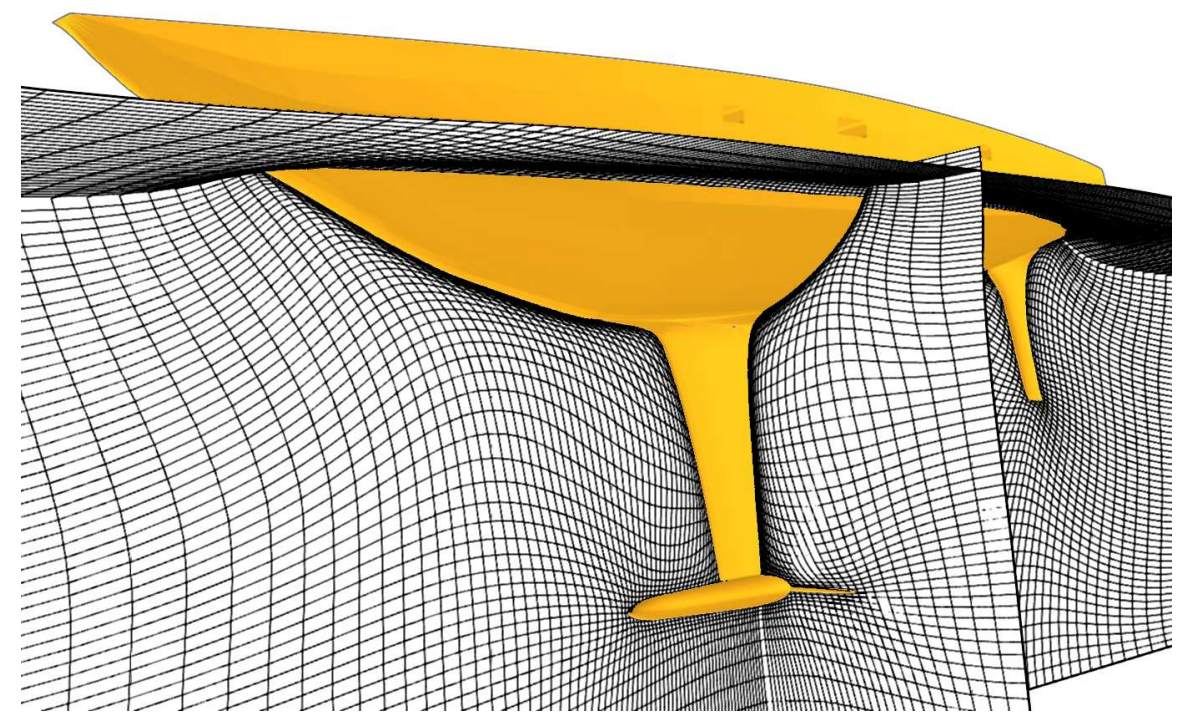

Figure 5.2: Deformed grid for computing the flow around a yacht (taken from Miemczyk [21]) 


\section{References}

[1] Thomas Apel, Sergei Grosman, Peter K. Jimack, and Arnd Meyer. A new methodology for anisotropic mesh refinement based upon error gradients. Appl. Numer. Math., 50(3-4):329-341, 2004.

[2] Ch. Becker, S. Kilian, and S. Turek. Hardware-oriented numerics and concepts for PDE software. In FUTURE 1095, pages 1-23. Elsevier, 2003. International Conference on Computational Science ICCS2002, Amsterdam.

[3] H. Blum. Asymptotic error expansion and defect correction in the finite element method. Habilitationsschrift, Universität Heidelberg, Im Neuenheimer Feld 294, 1991.

[4] P. B. Bochev, G. Liao, and G. C. de la Pena. Analysis and computation of adaptive moving grids by deformation. Numerical Methods for Partial Differential Equations, 12:489ff, 1996.

[5] J. U. Brackbill and J. S. Saltzman. Adaptive zoning for singular problems in two dimensions. J. Comput. Phys., 46:342-368, 1982.

[6] X.-X. Cai, D. Fleitas, B. Jiang, and G. Liao. Adaptive grid generation based on least-squares finite-element method. Computers and Mathematics with Applications, 48(7-8):1077-1086, 2004.

[7] W. Cao, W. Huang, and R. D. Russell. A study of monitor functions for twodimensional adaptive mesh generation. SIAM Journal on Scientific Computing, 20(6):1978-1994, 1999.

[8] W. Cao, W. Huang, and R. D. Russell. A moving mesh method based on the geometric conservation law. SIAM Journal on Scientific Computing, 24(1):118-142, 2002.

[9] W. Cao, W. Huang, and R. D. Russell. Approaches for generating moving adaptive meshes: location versus velocity. Applied Numerical Mathematics, 47:121-138, 2003. DOI: $10.1016 / \mathrm{S} 0168-9276(03) 00061-8$.

[10] G. F. Carey. Computational Grids: Generation, Adaptation, and Solution Strategies. Taylor und Francis, 1997.

[11] B. Dacorogna and J. Moser. On a partial differential equation involving Jacobian determinant. Annales de le Institut Henri Poincaré, 7:1-26, 1990.

[12] L. Formaggia and S. Perotto. Anisotropic error estimates for elliptic problems. Numerische Mathematik, 94:67-92, 2003. DOI 10.1007/s00211-002-0415-z.

[13] M. Grajewski. A new fast and accurate grid deformation method for r-adaptivity in the context of high performance computing. PhD thesis, Technische Universität Dortmund, Logos Verlag, Berlin, 2008.

[14] M. Grajewski and S. Turek. Establishing a new grid deformation method as tool in $r$ and $r h$-adaptivity. in preparation, Dortmund Technical University, Vogelpothsweg 87, 44227 Dortmund, 2008. 
[15] M. Grajewski and S. Turek. Practical aspects of a new fast and efficient multilevel grid deformation method. in preparation, Dortmund Technical University, Vogelpothsweg 87, 44227 Dortmund, 2008.

[16] E. Hairer, S. Norsett, and G. Wanner. Solving Ordinary Differential Equations I, volume I - Nonstiff Problems. Springer, Berlin, 1987.

[17] J. Lang. Adaptive Multilevel Solution of Nonlinear Parabolic PDE Systems. Springer, Berlin, 2000.

[18] G. Liao and D. Anderson. A new approach to grid generation. Applicable Analysis, 44:285-298, 1992.

[19] G. Liao and B. Semper. A moving grid finite-element method using grid deformation. Numerical Methods for Partial Differential Equations, 11:603-615, 1995.

[20] F. Liu, S. Ji, and G. Liao. An adaptive grid method and its application to steady Euler flow calculations. SIAM Journal on Scientific Computing, 20(3):811-825, 1998.

[21] Miemczyk. M. Hexaeder-Gittergenerierung durch Kombination von Gitterdeformations-, Randadaptions- und "Fictitious-Boundary"-Techniken zur Strömungssimulation um komplexe dreidimensionale Objekte. http://www.mathematik. uni-dortmund.de/lsiii/static/schriften_ger.html. Diploma thesis, Universität Dortmund, 2007.

[22] R. Panduranga. Numerical analysis of new grid deformation method in threedimensional finite element applications. http://www.mathematik.uni-dortmund. de/lsiii/static/schriften_ger.html. Master thesis, Universität Dortmund, 2006 .

[23] D. Wan and S. Turek. Fictitious boundary and moving mesh methods for the numerical simulation of rigid particulate flows. J. Comput. Phys., (22):28-56, 2006. http://dx.doi.org/10.1016/j.jcp.2006.06.002.

[24] A. Winslow. Numerical solution of the quasi-linear Poisson-equation in a nonuniform triangle mesh. J. Comput. Phys., 1:149-172, 1967. 